\title{
Atypical Presentation of Infective Endocarditis: A Case Report
}

Arian Bethencourt ${ }^{2}$, Daylin Rodriguez ${ }^{1}$, Dalie Ortet ${ }^{3}$, Tony L Brown ${ }^{4}$, Nicholas A. Kerna ${ }^{1,5^{*}}$, Joseph Reimon ${ }^{6}$, Robert Hernan$\mathrm{dez}^{7}$

${ }^{1}$ College of Medicine, University of Science, Arts and Technology, Montserrat, British West Indies

${ }^{2}$ Internal Medicine Department, Kendall Hospital, USA

${ }^{3}$ Clinica Biblical Hospital, Costa Rica

${ }^{4}$ Harvard University, USA

${ }^{5}$ Department of Internal Medicine, Suriwongse Medical Center, Thailand

${ }^{6}$ Internal Medicine, Kendall Regional Hospital

${ }^{7}$ Transitional Year Director, Kendall Regional Hospital

"Corresponding author: Nicholas A. Kerna, College of Medicine, University of Science, Arts and Technology, 4288 Youngfield Street, Wheat Ridge, CO 80033, USA. Email: nicholas.kerna@usat.edu

Citation: Bethencourt A, Rodriguez D, Ortet D, Brown TL, Kerna NA, Reimon J, Hernandez, R (2018) Atypical Presentation of Infective Endocarditis: A Case Report. Cardiolog Res Cardiovasc Med 3: 132. DOI: 10.29011/2575-7083.000032

\section{Abstract}

The American Heart Association defines Infective Endocarditis (IE) or bacterial endocarditis as an infection caused by bacteria that enter the bloodstream and settle in the heart lining, heart valve, or blood vessel [1].

IE is considered the fourth most common life-threatening infection syndrome after sepsis, pneumonia, and intra-abdominal abscess. In 2010, IE was associated with 1.58 million disability-adjusted life years, or years of healthy life lost, as a result of death and nonfatal illness and impairment $[2,3]$.

The variability in clinical presentation of IE and the importance of early diagnosis require a diagnostic strategy that is prompt for disease detection and specific for its exclusion across all forms of the disease [2].

Keywords: Bacteremia; Emboli; Endocarditis; Hemorrhage; LOC Infective; Intraparenchymal; Septic

\section{Abbreviations}

\section{AAOx3} place, and time-date and day)

Alert, Awake, and Oriented (to person,

$\mathrm{B} / \mathrm{L}$

CTA

$\mathrm{ETOH}$

HEENT

IE

IV ABX

LE
Bilaterally

Computed Tomography Angiography

Alcohol

Head, Ears, Eyes, Nose, And Throat

Infective Endocarditis

Intravenous Antibiotics

Lower Extremity
Multicenter

Clinic

MRA

MRI

NSR

NVE

Picc

TTE

TEE

RUQ US

UE
Level of Consciousness

More Than One Medical Center or

Magnetic Resonance Angiogram

Magnetic Resonance Imaging

Normal Sinus Rhythm

Native Valve Endocarditis

Peripherally Inserted Central Catheter

Transthoracic Echocardiography

Transesophageal Echocardiography

Right Upper Quadrant Ultrasound

Upper Extremity 


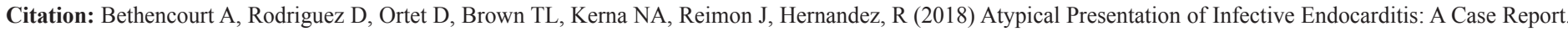
Cardiolog Res Cardiovasc Med 3: 132. DOI: 10.29011/2575-7083.000032

\section{Introduction}

The objectives of this case report are to increase awareness of the atypical presentations of IE, to emphasize the complications of IE, to avoid unnecessary invasive treatment for patients with septic emboli secondary to IE, and to promote timely diagnosis and treatment of patients with similar clinical presentations.

\section{Case Presentation}

We present a 68-year-old Caucasian male who reported left leg weakness during his intake interview at a local hospital. The patient had been experiencing leg weakness for one week. He was admitted to the hospital due to his complaint of leg weakness and fever.

After three days, he was discharged from the hospital with the impression of a viral infection. Subsequently, the patient's symptoms worsened. He returned to the hospital one week later and was readmitted due to his inability to walk and left side and left arm weakness.

The patient is married and living with his wife. He is a former ETOH abuser and former tobacco user (20 packs/year), and stated that he is currently a nondrinker and nonsmoker. The patient has worked for several years as a tugboat operator. The patient did not report any family medical history or known drug allergies.

\section{Medical History}

The patient reported a history of Hepatitis C, diabetes mellitus type 2, gastroesophageal reflux, aortic stenosis, and primary liver cancer metastasized to the right suprarenal gland. The patient had an aortic valve replacement one year ago, adrenalectomy (right), and chemoembolization about two years ago.

\section{Physical Examination Findings}

General Appearance: The patient was alert, awake, and oriented times 3 to person, place, and time. The patient did not display signs of acute distress.

Vital Signs: Temperature: $36.9^{\circ} \mathrm{C}$; blood pressure: $139 / 58 \mathrm{mmHg}$; heart rate: $83 \mathrm{bpm}$; respiratory rate: $19 \mathrm{rpm}$; O2 saturation $98 \%$.

HEENT: Normocephalic atraumatic; mucous membranes moist; extraocular muscles intact; pupils equally round and reactive to light, with accommodation bilaterally; no jugular venous distention.

Lungs: Clear to auscultation.

Heart: S1/ S2, holosystolic murmur at apex radiating to the axilla 3/6; no rubs, gallops.

Abdomen: Soft, nontender, nondistended.

Extremities: No edema; pedal pulses +; + Janeway lesions on the right palm.
Musculoskeletal: No joint swelling or deformity.

Skin: Venous stasis changes.

Neurological: AAOx3; Hand dominance: right.

Speech: Normal.

Memory: Short term/long term: intact.

Cranial nerves: 2 to 12 intact.

Sensory Exam: Normal: light touch, pin prick, position, vibration, temperature.

Motor testing 1: Normal: bulk, tone.

Motor Testing 2: The patient showed no asterixis, no dystonia, no fasciculation, no myoclonus, no tremor.

UE Left: $2 / 5$, UE Right: $5 / 5$

LE left: 1/5, LE Right: 5/5

Cerebellar Test: Absent nystagmus.

Present: Doll's eyes, corneal reflex, gag reflex.

Tendon Reflexes: 1+ B/L UE and LE

Plantar Reflexes: No response (right, left).

Gait: Not tested

Comments: The patient exhibited focal weakness, gait disorder. The patient denied bladder incontinence, bowel incontinence, change in LOC, confusion, dizziness, headache, lightheaded, numbness, seizure, slurred speech, spinning sensation, fainting, unable to speak, vision change, and generalized weakness.

\section{Laboratory Findings}

CBC: White count $14 \mathrm{mg} / \mathrm{dl}, \mathrm{H}$ and $\mathrm{H} 11 \mathrm{mg} / \mathrm{dl}, 35 \mathrm{mg} / \mathrm{dl}$, platelet count $61 \mathrm{mg} / \mathrm{dl}$.

CMP: Creatinine $1.16 \mathrm{mg} / \mathrm{dl}$, potassium 3.7Eq/l, alk phos 143U/I, $\mathrm{Mg} 2.3 \mathrm{mEq} / \mathrm{dl}$, Ca $8.2 \mathrm{mE} / \mathrm{dl}$, Pho $1.9 \mathrm{mE} / \mathrm{dl}$.

Coagulation: PT/INR: 17/1.6 sec; PTT: $31.5 \mathrm{sec}$.

HFP: Total bilirubin $2.1 \mathrm{mg} / \mathrm{dl} \mathrm{H}$; total protein $5.7 \mathrm{mg} / \mathrm{dl}$; AST 39 $\mathrm{U} / \mathrm{L}$, ALT $39 \mathrm{U} / \mathrm{L}$.

U/A: Negative.

EKG: NSR.

\section{Diagnostic Imaging}

Carotid doppler: Negative.

CTA: Intracranial hemorrhage, not aneurysm.

\section{CT head without contrast (below):}

$2.8 \times 3 \mathrm{~cm}$ right parietal parenchymal hemorrhage; right parietal and left parietal subarachnoid hemorrhage. 

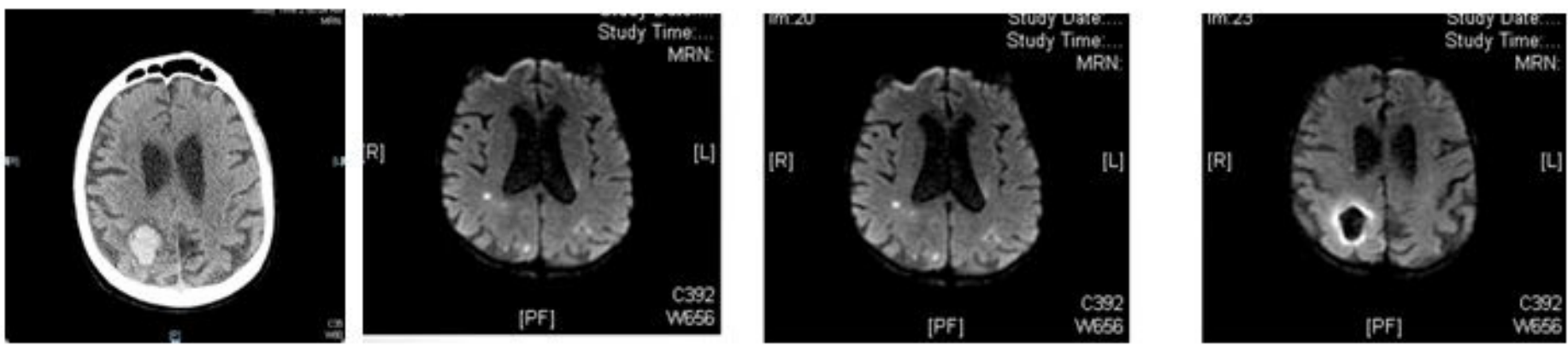

MRA: Technique throughout the carotid arterial system bilaterally showed calcified plaque at the carotid bulbs with no flow-limiting stenosis.

RUQ US (below): Mildly echogenic liver (possibly secondary to fatty infiltration); prominent portal vein.

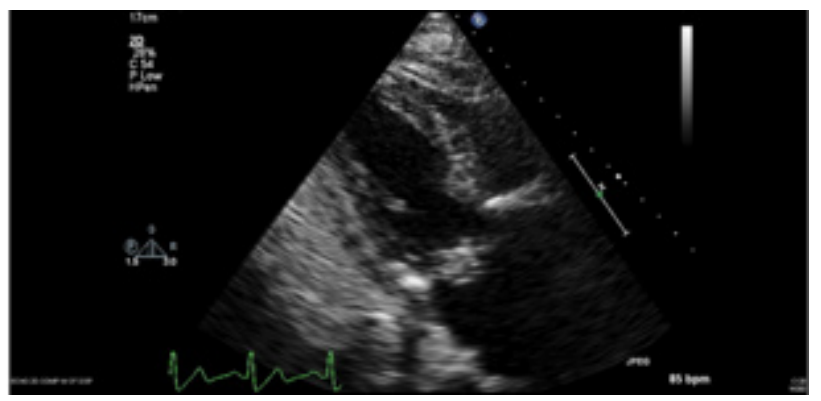

(This raised the consideration of early portal hypertension. It was suggested to correlate further with clinical findings.)

TEE: Transesophageal echocardiography (below).

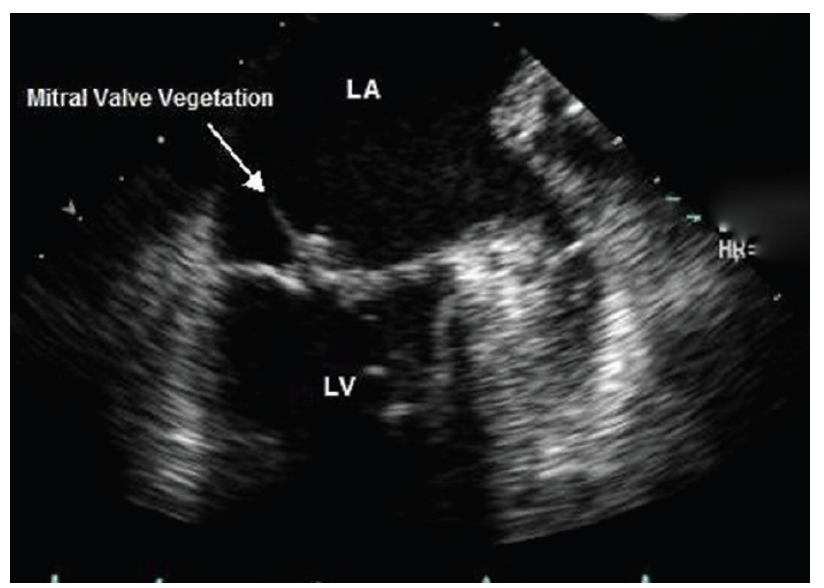

(Note. Image of mitral valve vegetation as shown on TEE from najms.org.)

\section{Assessment and Diagnosis}

The initial findings led to an impression of metastatic disease. After further evaluation and testing (including blood and urine cultures), the patient was given a definitive diagnosis of infective endocarditis.

It is important to note that the subsequent testing revealed two significant findings that suggested infective endocarditis by Duke criteria. (Major Duke criteria: positive blood culture tested two times, Gram-positive cocci in pairs as Streptococcus, and vegetation on the mitral valve noted on the $2 \mathrm{D}$ echo $10 \mathrm{~mm}$; Minor Duke criteria: Murmur, systolic murmur on the apex MR, and hemorrhagic stroke [4].) The patient suffered acute hemorrhagic CVA secondary to septic cardioembolism.

\section{Management and Treatment}

1. Administration of vancomycin empirically [5].

2. Blood culture taken twice.

3. Picc line for long-term IV ABX, 6 weeks.

4. Reevaluate for possible mitral valve surgery.

\section{Case Discussion}

Preliminary impression for the patient was a large intraparenchymal hemorrhage likely secondary to metastatic disease since the patient had a history of hepatic cancer versus coagulopathy thrombocytopenia.

During day 1 and 2 of treatment, the patient's MRI showed sizeable dominant hemorrhage in the right posterior parietal lobe demonstrating peripheral enhancement-which can be seen in metastatic disease but can also be due to the subacute phase of hemorrhage/ischemia. The findings showed large intraparenchymal hemorrhage, likely secondary to septic emboli. These findings contributed to the impression of metastatic disease, but other conditions were considered possible.

The care providers discussed differential diagnoses for amyloidosis (less likely because the bleeding area), an aneurysm, AV malformation, and Traumatic Brain Injury (TBI); however, the 


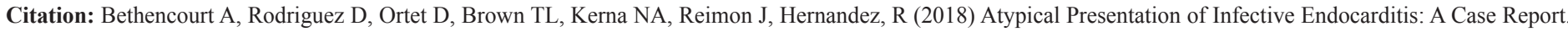
Cardiolog Res Cardiovasc Med 3: 132. DOI: 10.29011/2575-7083.000032

patient did not report any trauma. Approximately day 4, the care providers suspected IE.

On day 4 of treatment, an echocardiogram was ordered. An annular calcification in the mitral valve was observed as was mild thickening and normal leaflet separation and possible, mobile vegetation measuring $10 \mathrm{~mm}$ × $26 \mathrm{~mm}$. The transmitral velocity was within normal range. There was moderate regurgitation and no evidence of stenosis.

After reviewing the additional findings, an Infectious Disease (ID) inter-consult was completed. Blood and urine cultures were taken. The blood culture was positive for Gram-positive cocci (Enterococcus faecalis). TTE and TEE confirmed mitral valve vegetation. The definitive diagnosis was determined as bacterial endocarditis (2 major Duke criteria) with septic embolic and hemorrhagic bleeding.

The management of the condition was focused on continuing patient treatment with vancomycin, ceftriaxone, and ampicillin/ sulbactam (Unasyn); IV for 6 weeks.

The patient was discharged from the facility with close followup by the ID internist (infection disease specialist), a cardiologist, and a cardiothoracic surgeon. Mitral valve replacement was not needed. However, it was recommended to continue monitoring the bacteremia to assess the need for possible heart surgery and removal of the prosthetic valve.

\section{Considerations Regarding IE}

Complications of IE include cardiac, neurologic, renal, musculoskeletal; and complications related to systemic infection, such as embolization, metastatic infection, and mycotic aneurysm. These complications can co-occur and can be considered based on pathogenesis; such as cerebral infarct (embolic), heart valve destruction (the local spread of infection), vertebral osteomyelitis (metastatic infection), and glomerulonephritis (immune-mediated damage).

It has been researched and described that IE should be considered a possible etiology if the patient displays symptoms of systemic arterial embolization. The likelihood of IE is increased in patients with cerebral and systemic arterial embolization [6,7].

Echocardiography is central to the diagnosis and management of patients with IE. Evidence of an oscillating intracardiac mass or vegetation, an annular abscess, prosthetic valve partial dehiscence, and new valvular regurgitation are significant criteria in the diagnosis of IE [1]. Definitive diagnosis of IE can be made based on clinical manifestations, blood cultures, and echocardiography. Additional evaluations for patients with suspected IE include electrocardiography, chest radiography, and other radiographic imaging targeting clinical manifestations [8].

\section{Suggested Guidelines for the Timely Assessment and Treatment of IE}

During an intake interview, it is essential to assess a patient with a prosthetic heart device, a history of surgeries, or recent dental procedures. It is imperative to highlight cerebral and systemic arterial embolization as related complications of IE.

Echocardiography should be performed directly in patients suspected of IE (Class I; Level of Evidence A) [1].

At least three sets of blood cultures obtained from different venipuncture sites should be obtained, with the first and last samples drawn at least one hour apart. (Class I; Level of Evidence A) [1].

TEE should be performed if initial TTE images are negative, or inadequate in patients for whom there is an ongoing suspicion for IE, or when there is a concern for intracardiac complications in patients with an initial positive TTE (Class I; Level of Evidence B) [1].

If there is high suspicion of IE despite initial negative TEE, then repeat TEE is recommended in 3 to 5 days, or sooner if clinical findings change (Class I; Level of Evidence B) [1].

Repeat TEE after initial positive TEE if clinical features suggest a new development of intracardiac complications (Class I; Level of Evidence B) [1].

It is fundamental to obtain at least two sets of blood cultures every 24 to 48 hours until the bloodstream infection has cleared (Class IIa; Level of Evidence C) [1]

If operative tissue cultures are positive, then an entire antimicrobial course is indicated after valve surgery (Class IIa; Level of Evidence B) [1].

The risk of embolization in the setting of native valve endocarditis is 13 to 44 percent, and in many cases embolization occurs before a diagnosis of IE has been established. Among patients with IE, risk factors for embolization include vegetation size $>10 \mathrm{~mm}$; vegetation mobility; vegetation location on the anterior mitral leaflet; prior embolization; and infection with Staphylococcus aureus, Streptococcus bovis, or fungus.

In a multicenter study of 384 patients with definitive IE (75 percent with native valve endocarditis), S. aureus and $S$. bovis were predictors of total embolic events, while vegetation length $>10 \mathrm{~mm}$ and severe vegetation mobility were significant predictors of embolic events after initiation of antibiotic therapy. It is clear that the incidence of septic emboli in the setting of infected endocarditis depends on the size of the vegetation and the type of infective microorganism [9].

\section{Considerations for Early Valve Surgery}

Early valve surgery in left-sided NVE (during initial 


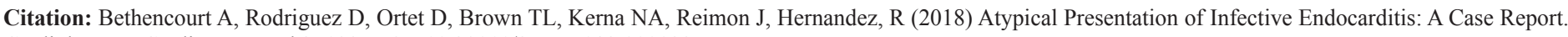
Cardiolog Res Cardiovasc Med 3: 132. DOI: 10.29011/2575-7083.000032

hospitalization and before completion of a full course of antibiotics) is indicated in patients with IE who present with valve dysfunction resulting in symptoms or signs of heart failure (Class I; Level of Evidence B) [1].

Early surgery should be considered, particularly in patients with IE caused by fungi or highly-resistant organisms; such as vancomycin-resistant Enterococcus or multidrug-resistant Gramnegative bacilli (Class I; Level of Evidence B) [1].

Early surgery is indicated in patients with IE complicated by heart block, annular or aortic abscess, or destructive penetrating lesions (Class I; Level of Evidence B) [1].

Early surgery is indicated with evidence of persistent infection after the start of appropriate antimicrobial therapy (Class I; Level of Evidence B) [1].

Early surgery is reasonable in patients who present with recurrent emboli and persistent or enlarging vegetations despite appropriate antibiotic therapy (Class IIa; Level of Evidence B) [1].

Early surgery is rational in patients with severe valve regurgitation and mobile vegetations $>10 \mathrm{~mm}$ (Class IIa; Level of Evidence B) [1].

Early surgery may be considered in patients with mobile vegetations $>10 \mathrm{~mm}$, particularly when involving the anterior leaflet of the mitral valve and other relative indications for surgery (Class IIb; Level of Evidence C) [1].

Valve surgery may be considered without delay in IE patients with stroke or subclinical cerebral emboli and residual vegetation if imaging studies have excluded intracranial hemorrhage and if neurological damage is not severe (Class IIb; Level of Evidence B) $[1]$.

In patients with major ischemic stroke or intracranial hemorrhage, it is recommended to delay valve surgery for at least four weeks (Class IIa; Level of Evidence B) [1].

\section{Conclusion}

Many complications are commonly observed in patients with infective endocarditis. Even though cardiac complications are the most common, neurologic and septic complications are the leading cause of death in patients with infective endocarditis [5]. The advantages of timely diagnosis and treatment for this patient-and patients with similar histories and presentations-include avoiding unnecessary and invasive interventions, and possible death. By presenting this case report, it is proposed that care providers can more readily recognize infective endocarditis and its atypical presentation in similar settings and circumstances.

\section{Conflict of Interest Statement}

The authors declare that this paper was written in the absence of any commercial or financial relationships that could be construed as a potential conflict of interest.

\section{References}

1. Infective Endocarditis (2018) American Heart Association, USA.

2. Baddour LM, Wilson, Walter R, Bayer, Arnold S, et al. (2015) Infective Endocarditis in Adults: Diagnosis, Antomicrobial Therapy, and Management of Complications. American Heart Association. Circulation $1-50$

3. Murray CJ, Vos T, Lozano R. Naghavi M, Flaxman AD, et al. (2012) Disability-adjusted life years (DALYs) FOR 291 diseases and injuries in 21 regions, 1990-2010: a systematic analysis for the Global Burden of Disease Study 2010. Lancet 380: 2197-2223.

4. Durack DT, Lukes AS, Bright DK (1994) New criteria for diagnosis of infective endocarditis: utilization of specific echocardiographic findings. Duke Endocarditis Service. Am J Med 96: 200-209.

5. Sexton, Daniel J, Calderwood, Stephen B (2018) Antimicrobial therapy of native valve endocarditis.

6. Pruitt AA, Rubin RH, Karshmer AW, Duncan GW (1978) Neurologic complication of bacterial endocarditis. Medicine (Baltimore) 57: 329

7. Mohr JP, Caplan LR, Melski JW, Goldstein RJ, Duncan GW, et al. (1978) The Harvard Cooperative Stroke Registry: a prospective registry. Neurology 28: 754-762.

8. Sexton, Daniel J, Fowler, Vance G (2018) Clinical manifestation and evaluation of adults with suspected native valve endocarditis. Circulation.

9. De Castro S, Magni G, Beni S, Cartoni D, Fiorelli M, et al. (1997) Role of transthoracic and transesophageal echocardiography in predicting embolic events in patients with active infective endocarditis involving native cardiac valves. Am J Cardiol 80:1030-1034. 